\title{
FORCE-DIRECTED SCHEDULING IN AUTOMATIC DATA PATH SYNTHESIS
}

\author{
P.G. PAULIN ${ }^{2}-$ J.P. KNIGHT ${ }^{2}$ \\ 1 Dept. 5L40, Bell-Northern Research \\ P.O.Box 3511, Stn C, Ottawa, ONT. K1Y $4 \mathrm{H} 7$ \\ 2 Carleton University, Colonel By Dr, Ottawa, ONT. K1S 5B6
}

\section{ABSTRACT}

The HAL system performs data path synthesis using a new scheduling algorlthm that is part of an interdependent scheduling and allocation scheme. This scheme uses an est1mate of the hardware allocation to guide and optimize the schedul 1ng subtask. The allocation information includes the number, type, speed and cost of hardwaro modules as well as the associated multiplexer and interconnect costs.

The iterative force-directed scheduling algorithm attempts to balance the distribution of operations that make use of the same hardware resources:

- Every feesible control step assignment is cvaluated at each iteration. for all operations.

- The assoclated side-effects on all the predecessor and successor operations are taken into account.

All the decistons are global

- The algortthm has $O\left(n^{2}\right)$ complexity.

Wo revicw and compare extsting scheduling techntques. Moderate and difflcult examples are used to illustrate the ef fectiveness of the approach.

\section{INTRODUCTION}

In the automatic design of application specific integrated circuits, the algorithmic description is commoniy synthesized into a datapath and a control path. Scheduling dacostions into the best control steps is a task datapath operations has been recognized in many systems whose tmportance has been recognized in many systems single most important step during the architecture synthesis". Ironicaliy, it is also the one that has received the least attention in current 1 fterature. (Notable exceptions are the papers presented by Alice Parker's group at the Untversity of Southern California $[2,3]$ and by Emll Girczyc of the University of Alberta [4]).

Operation scheduling determines the serial/parallel nature of the design and approximates cost-speed trade-offs [6]. If the design is subjected to a speed constraint, the scheduling algor ithm will attempt to make suffictent operations run in parallel to meet the constraint. Conversely. if there is a limit on chip area, the scheduler can be asked to sertalize operations to give the maximum speed consistent with the canstraint.

The scheduling task is an important one as it affects four fundamental aspects of the subsequent synthes is process:

This research was funded in part by grants from the $\mathrm{Na}-$ tural Sctences and Engineer ing Research Counc ll, Canada (NSERCC) and from Bel1-Northern Research, Ottawa.

Permission to copy without fee all or part of this material is granted provided that the copies are not made or distributed for direct contmercial advantage, the ACM copyright notice and the title of the publication and its date appear, and notice is given that copying is by permission of the Association for Computing Machinery. To copy otherwise, or to republish, requires a fee and/or specific permission.
- The number and type of processors allocated

- The timing constraints on these processors

- The storage requirements

The data transfer requirements

The HAL system is composed of three main modules that were described in [5]. The InHAL module uses a novel forcedirected scheduling algorithm that attempts to balance the distribution of operations that make use of the same hardware resources. This algarithm also allows specific allocation information, such as the propagation delay of a specific standard cell, to be used when optimizing the scheduling process. The MidHAL module performs the hardware al location while the final interconnection and optimization step is realized by the ExHAL module.

We will first review existing scheduling techrifques and compare them with the approach used in the HAL system. This will be followed by a description of the force-directed scheduling algorithm that is the main emphasis of this pascheduling algorithm that is the main emphasis of this paper. We will then demonstrate how processor allocation in-
formation is integrated into the scheduling process. finally, we present experimental results for moderate and difficult problems taken from current 1 iterature.

\section{LITERATURE SURVEY}

The stmplest way to perform scheduling is to relegate the task to the user. This is the approach favored by the stlc system [7] under the assumption that the user should explicitly define the parallelism of the design.

\section{Independent scheduling/allocation schemes:}

The next simplest scheme is to schedule aperations "as soon as possfble" (ASAP) as is done in the Emerald/Facet system [8]. This technique has proved useful in the past for near optimal intcrocode compaction [9].

A refinement of this concept is ASAP scheduling with conditional postponement of operations. In the MIMOLA system [10], this occurs whenever the operation concurrency is higher than the number of avaliable processors. The recently published $F$ lamel system [17] uses the same ldea. The scheme published Flamel system [17] Uses the same Idea. The scheme
used in Kung. Whitehouse and Kallath's book on digltal signal processing [11] is similar, except that an operation is postponed when it blocks a later one with a lower "as late as possible" (ALAP) level.

Continuing along the scale of increasing complexity, we have the algorlthms that use 11 st scheduing. as described in [9]. The behavioral synthesis of interfaces (BSI) system developed by Nestor [12], the SLICER system developped by Pangrie and Gajsk 1 [13] and the BUD-DAA system [14] use this type of approach.

In list scheduling, operations are sorted in topological or der (top to bottom) by using the precedence relations alctated by data and control dependencies. The sorted operations are then iteratively scheduled into contro? steps. The order in which they are placed into a control step is determined by a heuristic priority function that is applied to all operations that can be placed in the control step.

In the BSI system, the priority function reflects whether placing the operation in the current step will violate a minimum time constraint (ASAP time) and whether placing an operation in a later step will violate a maximum time constraint (ALAP time). 
In the SLICER system, the priority function is based on operation moblifties. The mobility of an operation is defined as the difference between it's ASAP time and ALAP time. Operations on the critical path are thus scheduled first.

The BUD-DAA system uses a simflar priority function but sorts the operations in depth-first order (bottom to top).

Strictly speaking, the scheduling is not totally independent of the allocation in these systems. Most derive estimates of the operation delays based on the most likely processor assignments.

\section{Interdependent schedul ing/al locat ton schemes:}

In these systems, the operation scheduling is done concurrently with the processor allocation (except for the Stic system, where the user has final say). Elf uses a varlation system, Where the user has firm sayl. the priority function of the list scheduling algorithm where the priority function is based on operation wetghts and urgencles. Operation weights are calculated by taking the minimum number of cysuccessors. Operation urgencies are determined by taking ratio of the operation's weight and the number of cycles left until its time constraint. When an operation is delayed its urgency increases which raises it's priority for assignment in the next control step.

The elaborate scheme used in USC's MAHA system [2] relies on critical path determination and the concept of operation freedom to guide scheduling. The freedom of an operation is identical to the mobility calculated in the SLICER system described earlier.

The MAHA system first invokes the clocking Scheme Synthesis Package (CSSP) written by Park [3]. Here the critical path is determined and divided optimally into $n$ steps, one per clock cycle. The clock cycle is also automatically deter mined nere. In the HAL system, the clock cycle must be defined by the user.

MAHA then allocates processors for the critical path in first-come first-served fashion. The notion of freedom is used to guide the scheduling of the off-critical-path nodes. The node with the smallest freedom is chosen for allocation. The critical path astgrment is completed in 1 inear $t$ ime The assignment of off-critical-path nodes is done in $O\left(n^{4}\right)$ t ime in the worst case.

We can thus summarlze the approaches:

- Independent scheduling/al location

- ASAP scheduling approaches:

- Direct (Facet-Emarald)

Conditional deferment (MIMOLA, Flamel, [11])

- List scheduling approaches:

- Priorlty function: time constraints (BSI)

- Priority function: mobility (SLICER)

Priority function: critical path

use of depth-first ordering (BUD-DAA)

- Interdependent scheduling/allocation

- User-defined schedule (silc)

- List scheduling using urgency (ELF)

- Freedon and critical path scheduling (MaHA)

- Force-directed scheduling (HAL)

\section{SYSTEM COMPARISON}

The HAL system's approach to scheduling is fundamentally different from all of the approaches discussed above. We describe here three of the most important differences.

\section{Local ve qlobal evaluations:}

This is, in our view, the most important novel contribution of the HAL system to the scheduling/allocation task. Virtually all the systems discussed rely on a local evaluation of the effect of the control step assignment of the current operation constdered.

The HAL system considers the related effects of a control step assignment on all the predecessor and successor operat Ions. Moreover, the assignment's impact on the overall
concurrency of operations is evaluated through the use of distribution graphs (DG). These DGs represent the current concurrency of each class of operation at each control step (c-step from now on). Unscheduled operations also contribute to the determination of the operation concurrency except ute to the determination of the operation concurrency except deterministic.

Finally, every feaslbie assignment of each operation to any of its possible c-steps is considered at each iteration.

While this global approach would seem to imply large computation costs, the algorithm is $O\left(n^{2}\right)$, where $n$ is the number of operations. This relatively low order of complexity is due to an efficient method of propagating predecessor and successor forces. We shall give a brief description of this method in section " 4.3 ORDER OF COMPLEXITY".

\section{Independent vs interdependent schedul ing/al locat ion:}

In the Facet, MIMOLA, BSI, SLICER and BUD-DAA systems the scheduling is performed more or less independently of the
processor allocation. The Elf and MAHA systems do both tasks concurrently.

The HAL system, on the other hand, does both tasks separately but not independently. The operation scheduling and processor allocation are determined by stepwise refinement as depicted in Figure 8 of section " 5 . SCHEDULING/ALLOCATION BY STEPWISE REFINEMENT".

\section{Topologtcal vs middle-out schedul ing:}

The final difference relates to the order of selection of operations during the scheduling process. In all the systems mentioned above (with the exception of MAHA), the operations are scheduled iteratively by using their topological order (depth-first order in BUD). The MAHA systopological order (depth-first order in BUD). The MAHA system first schedules the operations on the crftical path and
then schedules the remaining ones in order of increasing freedom.

In the HAL system, the ordering is based on the lowest force. As we shall see in the next section, this value is independent of the precedence ordering. It may be considered a 'middle-out' approach.

\section{FORCE-DIRECTED SCHEDULING ALGORITHM}

The selection of the operation to be scheduled, and of the c-step to which it włll be assigned, is based on an evaluation of the move which causes the most balanced distribution of operations in each c-step. This algortthm is an t terative one in which one operation is scheduled at each iteration. The intent is to reduce the number of processors required by reducing the concurrency of the operations assigned to them, but without lengthening the total execution time

\subsection{BASIC SCHEDULING ALGORITHM}

\section{Determination of time frames:}

The first step involves the determination of both an ASAP (as soon as possible) scheduling and an ALAP (as late as possible) scheduling. Combining results for both schedules w11] determine the possible time frames for each operation. To ilustrate this, we will use the example given in [5]. The DFG derived from this example is given in Figure 1 . The DFG derived from this example is given in Figure 1 . (control steps). Here. ASAP scheduling ts shown. For simplicity, it will be temporarily assumed that all operations require one clock cycle and that succeeding operations cannot be scheduled in the same cycle.

The ASAP and ALAP operation scheduling are shown (in simplified form) in Figure 2. 

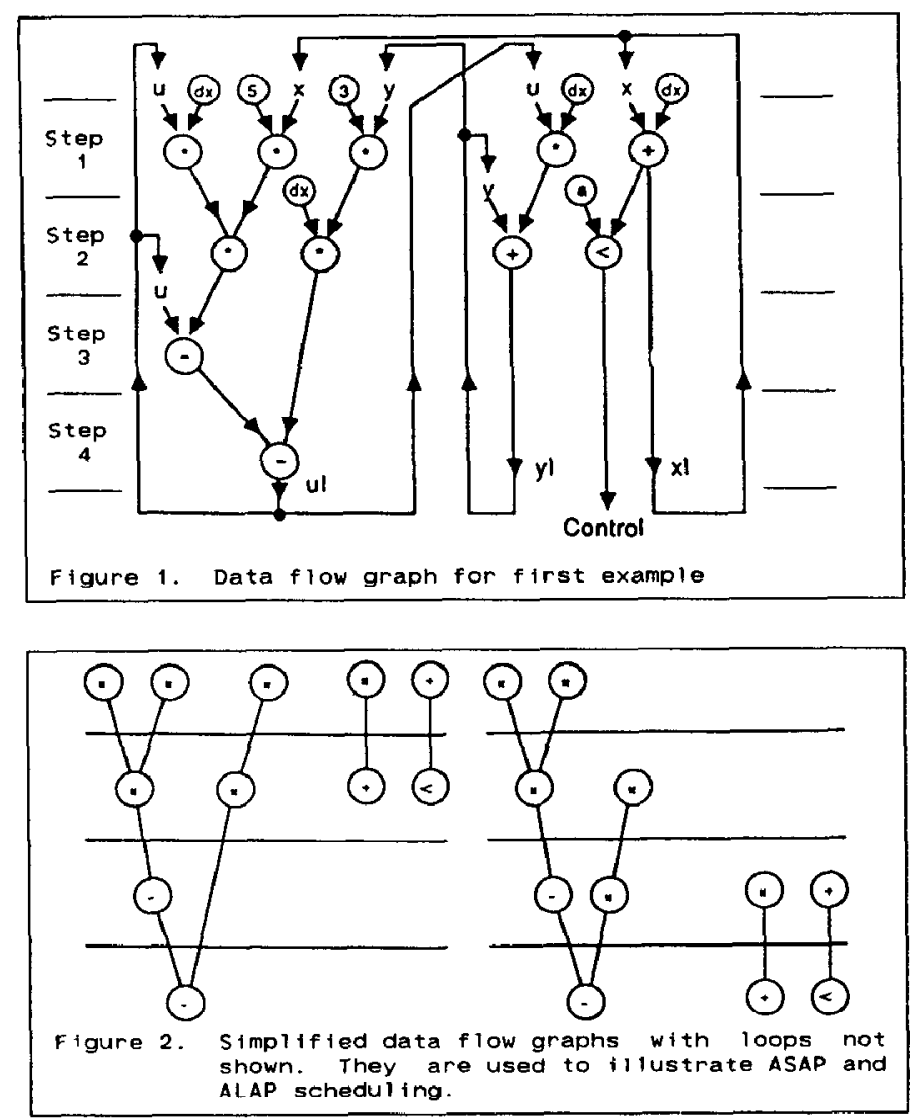

By noting that the actual scheduling of a particular operation may be anywhere between it's ASAP cycle and it's ALAP cycle, one can draw a time frame diagram (Figure 3 ). Here the width of the box containing a particular operation represents the probability that the operation will be eventually placed in a given time slot. A useful heuristic is to assume uniform probability of placing an operation in any feasible control step. The area of each operation is always one, but $1 \mathrm{t}$ is 'stretched' along its time frame.

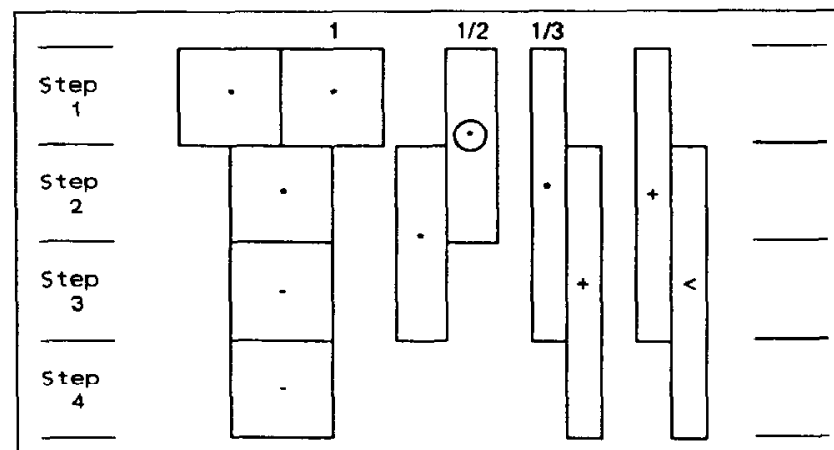

Figure 3. Time frames of operations (1nitlal state).

\section{Craction of diatributton oraphs (DGg):}

The next step is to take the summation of the probabilities of each type of operation for each control step of the DFG the resulting distribution graphs (one for each type of opThe resulting distribut ion graphs (one for each type of op-
eration) indlcates where concurrency of similar operations is high and will direct the scheduling algorithm accordingly.

\section{Condittonal statements:}

The presence of conditional stalements (e.g. If-then else and case statements) causes some operations to be mutualiy and case statements) causes some operations to be mutualiy exclusive. When these operations can be executed on the
same processor type (1.e. assigned to the same DG), they can be scheduled into the same c-step without increasing the processor allocation. The same processor ts simply shared by those operations as they will never be executed concurrentiy.

We use the following stratagem to take advantage of this observation: ror each c-step in whtch the time frames of the mutually exclusive operations intersect the probability of only one of these is added to the corresponding DG. The operation selected is the one with the highest probablifty. This is 1llustrated for the simple DFG of Figure 4 . The fork and join operations correspond to an if-then-else statement.

Without special treatment of the mutually exclusive additions, the total distribution would be 1.5 in both c-steps. The unscheduled addition would then have an equal probabilthe unscheduled addition assigned to either c-step. It is obviousiy ity of being assigned to etther c-step. It is obviously preferable to schedule it in the first c-step, as in this
case only one adder will be required. This is exactly what will happen using the stratagem just descrlbed due to the reduced distribution in the first c-steo.

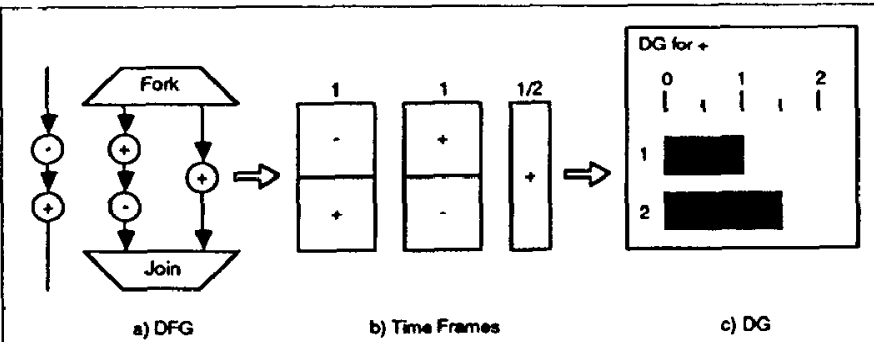

Figure 4. Distribution evaluation for DFG with conditional statement.

The distribution graphs derived from $F$ igure 3 are given below in Figure 5 .
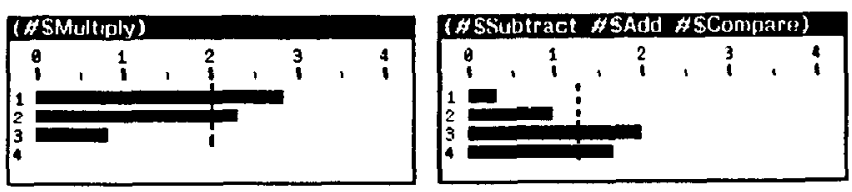

Figure $b$. Distribution graphs (initial state).

The first graph represents the distribution of the multiply operations. and the next one combines the distributions of the add, subtract and compare operations. The latter three operations are actually assigned to separate DGs but are grouped here for conciseness. Each horizontal bar of the DGs corresponds to a distinct c-step.

\section{Calculation of 'self' forces:}

Each operation of the DFG will have a force assoclated with each cycle of ti's time frame. This is a quantity which re* flects the effect of an attempted control step assignment on the overall operation concurrency. It is positive if the assignment causes an increase of operatton concurrency and negative for a decrease.

The force is much like that exerted by a sertes of 'springs' that obey Hooke's law: $F=K x$. K represents the spring's constant (rigidity), $x$ the displacement and $F$ the force caused by the displacement.

Each distribution graph will have assoclated springs (one for each c-step) that will exert forces on all operations, The constant of the spring $K$ is represented by the value of $D G(t)$ where i is the c-step number for which the force is calculated. The displacement of the spring $x$ is given by calculated. The displacement of the spring $x$ is given by the tncrease (or decrease) of the probabtitty of the opera
tion in the c-step due to a rescheduling of the operation.

We will illustrate this by using the partial time frame diagram of Figure 6 . The constant of each of the three springs corresponds to the value of the multiplifation DG given in Figure 5 for the first three c-steps.

We will attempt to schedule the ctrcled multiply operation in c-step 1 as depicted in Figure 6 b). The probability of the operation will change from $1 / 2$ to 1 in $c-s t e p 1$ and from 
$1 / 2$ to 0 in c-step 2 . These probabllity shifts correspond to the displacement $x$ of each of the 'springs'. The resulting force associated with the move to e-step 1 is the sum of forces in both c-steps and is given by:

$$
\begin{aligned}
\text { Force (1) } & =K \times(1)+K \times(2) \\
& =\operatorname{DG}(1) * x(1)+\operatorname{DG}(2) * \times(2)
\end{aligned}
$$

Using the values from Figure 5 and Figure 6 we obtain:

$$
\begin{aligned}
\text { Force }(1) & =(2.8333 * 0.5)-(2.3333 * 0.5) \\
& =+0.25
\end{aligned}
$$

The force is positive as expected because the concurrency in c-step 1 is higher than in c-step 2. Scheduling the multiplication in that c-step will have an adverse effect on the overall distribution.

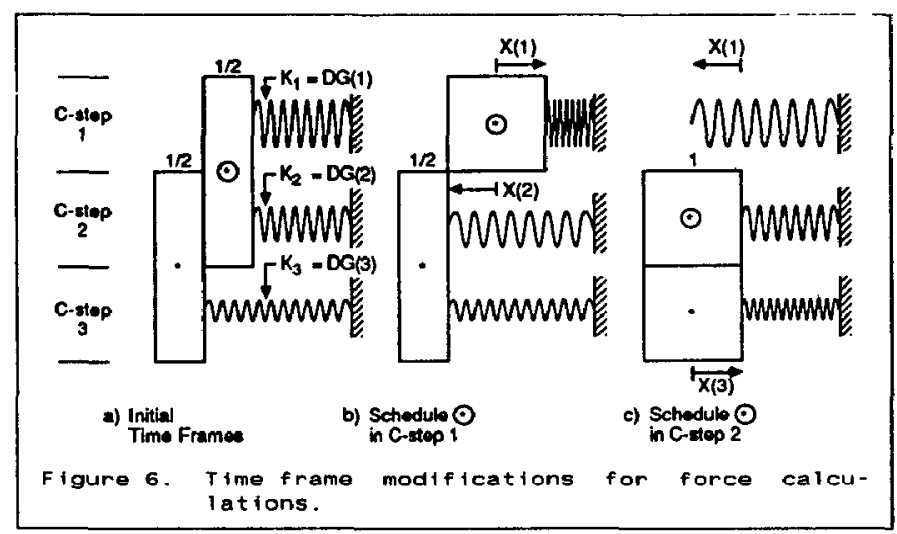

\section{Calculation of predecessor and successor forces:}

Assigning an operation to a specific c-step will often affect the time frames of linked operations in the DFG. In turn, this will create additional forces that can reduce or even counter the original intended improvement so it is imperative that they be accounted for

This is achieved by calculating the extra forces due to the tmplicit rescheduling of linked operations. They shall then be added to the 'self' force. There $w 11 l$ be two extra force contributions: the predecessor forces and the successor forces.

For example. If the circled multiply operation in Figure 6 a) was tentatively assigned to c-step two, as in Figure 6 c), the succeeding multiply operation would implicitly be assigned to c-step three. The time frames of the other operattons would on't be affected. The resulting force assac 1ated with that assignment would then be the sum of the two individual forces. The force of the first multiply ts given by:

$$
\begin{aligned}
\text { Force' (2) } & =(D G(1) * \times(1))+(D G(2) * \times(2)) \\
& =-(2.8333 * 0.5)+(2.3333 * 0.5) \\
& =-0.25
\end{aligned}
$$

The successor force of the second multiplication is given by:

$$
\begin{aligned}
\text { Force" (2) } & =(D G(2) * \times(2))+(D G(3) * \times(3)) \\
& =-(2.3333 * 0.5)+(0.8333 * 0.5) \\
& =-0.75
\end{aligned}
$$

And the resulting force of the c-step assignment is:

$$
\begin{aligned}
\text { Force (2) } & =\text { Force'(2) + Force" (2) } \\
& =-1
\end{aligned}
$$

This c-step asstgnment is much more advantageous than the one attempted earlier, and this is reflected clearly by the force calculations.

\section{Selection of best move:}

The self, predecessor and successor forces are calculated for each possible c-step of all the operations. The operation and c-step palr selected is the one with the most negative force (or the lowest positive force). The operation's time frame is then reduced to the selected c-step and the linked operations' time frames are modified accordingly.
We can thus summarize the entire process

1. Determination of time frames

2. Update of DGs (wtth conditionals taken inta account)

3. Calculation of 'self' forces

4. Calculation of predecessor and/or successor forces

5. Scheduling of best operation and c-step pair

These steps are repeated until the $t$ tme frame of each operation is reduced to one. The distribution graphs are updated at each iteration to reflect the current operation distributtons. The forces on the rematning unscheduled operations will vary accordingly.

\section{Sample scheduling:}

Figure 7 depicts the time frames and the final distributions for the example of Figure 1 .
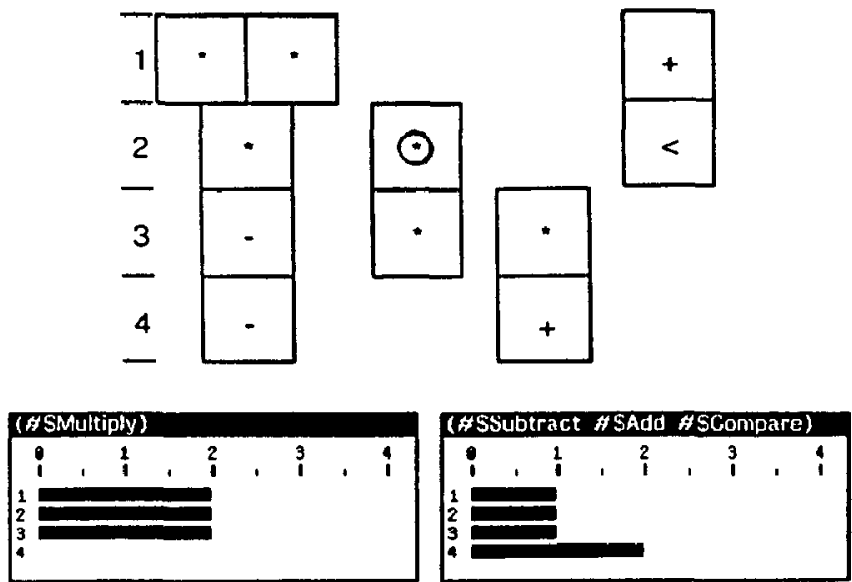

Figure 7. Final time frames and DGs

\section{2 REF INED SCHEDULING ALGORITHM}

\section{Mult1ple operation DGs:}

In many cases we have to deal with multi-function processors (e.g. Alus). To deal with this, the concept of distribution graphs (DG) was extended so that the distribution of one or more operation types can be stored in a single DG.

For example, If the allocator asstgns additions and subtracttons to an Alu, then a two class DG will be created in lieu of the two single class ones. These two types of operafions will then tend to be scheduied in different clock cycles to make best use of the Alu.

\section{Mult1ple operat ons per cycle and mult 1 -cycle operat tons:}

The possfbflity of scheduling muitiple dependent operations in a single cycle has also been incorporated to the system. in a single cycle has also been incorporated to the system. extending the time frames of fast combinatorial operations into the previous and/or next cycies (when the total delay in those cycles is less than the cycle time). Multi-cycle operattons are also supported and are implemented with a simple extension of the single-cycle methodology.

\section{Incorporation of processor costs:}

Different processor cells have different realization costs. Operation types associated with high-cost processor cells should be given higher priority in the scheduling process. An easy way to do this is to multiply the constant of the 'spring' associated with the OG by a cost factor that is a function of the processor area and the associated mux and interconnect costs. This. function will be given in section interconnect costs. This. function will be given in
" 5.3 USE OF ALLOCATION INFORMATION FOR SCHEDULING".

\section{Note on storage requirements:}

The minimum number of registers required for the implementation of a given DFG is given by the largest number of data arcs traversing a control step boundary.

Most of these data arcs are initiated by operation nodes. As the force-directed scheduling algorithm attempts to dis tribute these operations evenly across the DFG. the number of arcs traversing a given control step is also balanced. In turn. this reduces the minimum number of registers requ ired.

Paper 12.1

198 
For the ASAP scheduling shown in Flgure 1 at least seven registers are required (there are seven values to be stored at the end of cycle one). For the optimized scheduling shown below, this number is reduced to five (although in the final synthesized data path, six registers were used to save on interconnect).

Although these observations are encouraging, a more explicit treatment of register allocation w111 st 111 be required in the future. For more information on the HAL system's pres ent approach to register allocation, refer to [5]

\subsection{ORDER OF COMPLEXITY}

It can easily be seen that the algorithm described above is $o(n$ ') when implemented in a straightforward fashion ( $n$ is the number of operations). Fortunately, we have found an alternate method of including the forces of predecessor/successor operations that can be applied without any loss of generality. In this method, the self. predecessor and successor forces are calculated in three separate passes.

In the first pass the force applied directly to each operation ( 1 ts self force) is calculated for every c-step in its time frame. This calculation is done in linear time. These values are then stored in a vector assoctated with the operation.

In the second pass, the DFG is traversed in depth-first or der. The total force for each operation is given by the sum of its self force and the stored total force of its direct successors only. In this way we w1ll effectively be performing a running sum of all the successor forces in linear time. In the third pass the process is repeated for the predecessor forces by taking the operations in topological order.

Using this method, the order of complexity of the algorithm is now $0\left(n^{2}\right)$. This 15 accomplished at the expense of a sight increase in computer memory utilization.

\section{SCHEDULING/ALLOCATION BY STEPWISE REFINEMENT}

The scheduling/allocation iterative loop used in the HAL system attempts to reconclle two conflicting goals:

- The optimal scheduling of operations without explicit foreknowledge of the processor allocation.

The optimal allocation of processors without exact information on the concurrency of operations or their propagation delays.

This is accomplished by stepwise refinement of the operation scheduling and processor allocation as depicted in Figure 8 .

\section{1 SCHEDULING/ALLOCATION PHASES}

Phase 1 Default Allocation: Allocation of default singlePunction processors to perform each type of opera tion. The fastest processors are temporarlly as sumed.

Phase 2 Preliminary Schedule: Balancing of the distribution of similar operations. Default processor speed and area costs are used.

Phese 3 Refined Allocation: Allocation of single and multi-function processors with relaxed constraints on processor speed.

This can be done as it now has a better estimate of

- The timing constratnts on the individual operations (they may have been relaxed on some op-

- The overal1 concurrency of operations of different classes.

As deplcted in $F$ igure 8 , the mux and interconnect area costs are also evaluated here.

Phase 4 Final Schedule: Balancing of the distribution of operations requiring similar processor types. The processors are used here to gutde the scheduling.

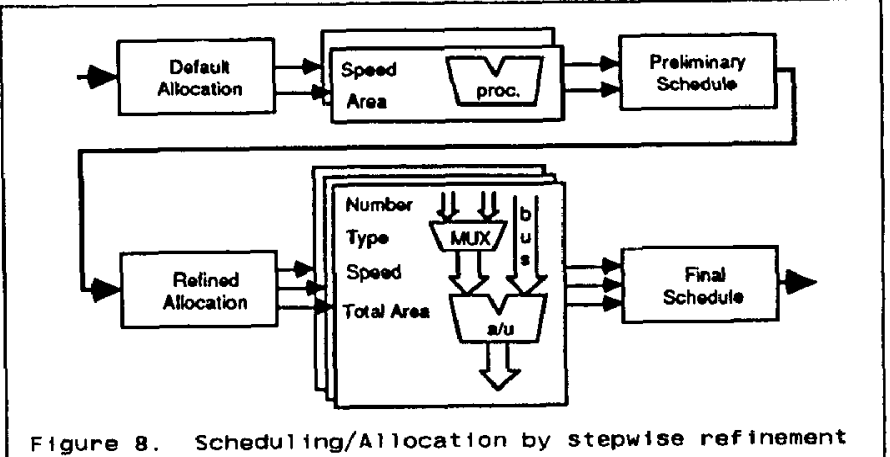

Before going on to describe how scheduling and allocation are linked, we will give a short description of the module (named MidHAL) that performs the allocation task. A more elaborate one is given in [5].

\subsection{THE MIDHAL ALLOCATER}

This module is 1 mplemented as a rule-based expert system. It accepts as input the DFG, a global ttme constraint, the local time constraints on each oporation and a library of avallable processors and their assoclated speed and cost. It then uses heuristic rules to select a set of locally optimum processors to execute the operations described in the DFG. given the time constraints on these operations.

The following information is returned:

- The type of processors allocated

- The number of processors of each type

- Their propagation delay

- Their area cost (including mux and triterconnect area)

The mux and interconnect area cost is evaluated by partitioning the DFG and doing a preliminary asstgmment of operations to processors. The data transfer requirements can then be evaluated. The mintmum number of multiplexers and interconnections required to perform these data transfers is then est imated.

The function and intent of thts module is quite similar to that of the BUD module in the recent BUD-DAA system [15]. However as preliminary floorplanning is not performed in the HAL system, the interconnect area estimation wlll not be as precise.

Moreover, the HAL system allows the allocation task to be performed completely or partially by the user. The system will verify that the allocated hardware is suffictent and will add processors as necessary. In this way the user can invoke the scheduling/allocation loop repeatedly with difinvoke the scheduling/alocation loop repeatediy with difsemi-automatically.

\subsection{USE OF ALLOCATION INFORMATION FOR SCHEDULING}

We shall show here how the four types of information returned by the MidHal module can be used to guide the scheduling process.

\section{Types of processors al located:}

For each type of procesor allocated, one single distribution graph (DG) is created. Multi-function processors such as scribed in sect ton "4.2 REFINED SCHEDULING ALGORITHM"

\section{Number of processors allocated:}

In most cases, the MidHAL module allocates the minimum number of processors for every type of operation. This number is equal to the maximum number of concurrent operations they perform. But there are also cases where a larger number of processors are allocated. This may happen when the cost saving due to processor sharing is offset by associated saving due to processor sharing costs. We must include this information so that the forces applied by the assigned DG are reduced, as more than enough processors are likely to be avat lable. 
More specifically, for each processor type (i.e. for each $D G)$ and each c-step we calculate the unused processor capacity. This is given as the difference between the number of allocated processors and the current number of operations scheduled in c-step 1

Unused Capacity $(1)$ = No Processors - No Opns( 1 )

The new biased DG value (i.e. the new spring constant) is then given by the old value less the unused processor capac-

Biased DG(i) = DG(i) - Unused Capacity(i)

When the number of scheduled operations in c-step 1 reaches the number of processors allocated, DG(i) will take on its or Iginal value.

\section{Processor speed:}

This information is integrated in a straightrorward fashion by assigning the propagation delay of each processor to the operations it performs. The determination of $t$ ime frames and subsequent scheduing will thus be directly affected by these new values.

\section{Processor, mult liplexer and interconnect costs:}

As we mentioned in section "4.2 REFINED SCHEDULING ALGORITHM" each DG element is multiplled by a cost factor. For the inttial scheduling in phase 2 , this cost factor is simply the processor area:

Cost Factor = Processor Area

For the final scheduling in phase 4 , we can use the est imate of the mux and interconnect area derived in phase 3 to calculated a weighed cost factor:

Cost Factor = Processor Area * Interconnect Factor

The interconnect factor represents the relative importance of the processor area with respect to the combined processor, mux and interconnect area:

Interconnect Factor $=\frac{\text { Processor Area }}{\text { Total Area }}$ where

Total Area $=$ (Processor + Mux + Interconnect) Area

For high-cost processors such as multipliers the interconnect factor tends towards one. Alternately. for simple processors (e.g. logic gates) the mux and interconnect area can be much larger than the processor area in which case the interconnect factor tends towards zero.

In general, this will result in an tncreased number of low cost processors, but the assoctated area increase will be offset by reduced multiplexer and interconnect costs. On the other hand, sharing of high-cost processors will still be other hand.

\subsection{SCHEDULING UNDER FIXED HARDWARE CONSTRAINTS}

The scheduling and allocation approaches presented above support the synthesis of near-mintmal cost datapaths under fixed timing constraints. A simple extension of this methodology also allows the determination of a schedule with a near-minimal number of c-steps given fixed hardware constraints.

The basic idea consists of performing regular force-directed scheduling with a ttght timing constraint. During the scheduling. hardware resource costs are estimated at every scheduling, hardware resource costs are est imated at every
t teration. Whenever a nardware constraint is exceeded (this tteration. Whenever a hardware constraint is exceeded (this
can be a constraint on the number of processors of any type, or a constraint on the total hardware cost), then an extra c-step is added to the graph. Thus the time frames of scheduled and unscheduled operations are extended by one $c$ step.

This increases the time required to arrive at a solution. but not the order of complexity of the algorithm. This is because the partial scheduling is maintained, f.e. the time frames of operations are extended, not recalculated.

This extra capability will be lliustrated in the example of section " 6.3 TEMPERATURE CONTROLLER FROM ELF".

\subsection{SAMPLE SCHEDULING AND ALLOCATIONS}

The processor allocations for the example given in section "4. FORCE-DIRECTED SCHEDULING ALGORITHM" are tabulated in Figure 9 for different time constraints. The refinements of the scheduling algorithm described above were applied nere. (Note: The multiplications are given a more realistic propagation delay of two cycles. The critical path is now six cycles long.) The cPu execution times given are for a XEROX 1108 Lisp machine.

\begin{tabular}{|c|c|c|c|c|c|c|c|c|}
\hline \multirow{2}{*}{$\begin{array}{c}\text { COMPONENT } \\
\text { No Cycles } \\
\text { multipl ler } \\
(*)\end{array}$} & \multicolumn{4}{|c|}{$(* \cos t>\operatorname{aru} \cos t)$} & \multicolumn{4}{|c|}{ (alu cost $>* \cos t)$} \\
\hline & $\begin{array}{c}6 \\
\cdots \\
(*) \\
(*) \\
(*)\end{array}$ & $\begin{array}{c}7 \\
-\cdots \\
(*) \\
(*)\end{array}$ & $8-12$ & \begin{tabular}{l}
13 \\
\hdashline-
\end{tabular} & \begin{tabular}{l}
$6^{\prime}$ \\
\hdashline$(*)$ \\
$(*)$ \\
$(*)$ \\
$(*)$
\end{tabular} & $\mid \begin{array}{c}7^{\prime} \\
--- \\
(*) \\
(*) \\
(*)\end{array}$ & $\begin{array}{r}8^{\prime}-12^{\prime} \\
-----\end{array}$ & $43^{\prime}$ \\
\hline $\begin{array}{c}\begin{array}{l}\text { alu } \\
(\mathrm{a})\end{array} \\
\mathrm{CPU}(\mathrm{sec})\end{array}$ & $\begin{array}{l}\text { (a) } \\
(\mathrm{a}) \\
--1- \\
15\end{array}$ & $\begin{array}{l}\text { (a) } \\
(\mathrm{a}) \\
-2 \\
35\end{array}$ & \begin{tabular}{c} 
(a) \\
\hdashline $65-120$
\end{tabular} & $\left(\begin{array}{l}(a) \\
--- \\
180\end{array}\right.$ & (a) & $\left|\begin{array}{c}(\mathrm{a}) \\
--- \\
30\end{array}\right|$ & $\begin{array}{c}\text { (a) } \\
-65-125\end{array}$ & $\mid \begin{array}{l}(\mathrm{a}) \\
-\infty \\
175\end{array}$ \\
\hline & & & $\begin{array}{l}\text { a } 11 \\
\text { EX }\end{array}$ & on & $F i c$ & 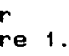 & a 11 & ime \\
\hline
\end{tabular}

The results in the first four columns 11 lustrate the real is tic case where the cost of the multiplich is higher than the cost of the Alu. The results in the next four columns represent an illustrative example where the cost factor of the Alu is set artificially higher than the cost of the multiplier. The change in forces resulted in the decrease of the concurrency of the Alu operations at the expense of the multiplication operations. This is exactly the trade-off that we are hoping for.

The results obtained in column $6^{\prime \prime}$ and $7^{\prime}$ are identical to the ones that would be obtained if the MidHaL module (the processor allocater) had allocated four and three multipliers respectively. This is due to the associated reduction of the multiplication forces dictated by equation (2) of section "5.3 USE OF ALLOCATION INFORMATION FOR SCHEDULING" . such that the concurrency of the Alu operations is reduced to one. Once again, this trade-off is the one that should follow logically.

\section{EXPERIMENTAL RESULTS/COMPARISONS}

The four examples presented in this section are taken from sothe of the systems described in section "2. LITERATURE SURVEY". They where chosen to illustrate the flexibility of the HAL system with respect to considerably different constraints and also to allow comparison with the results obtained from these systems.

\section{Experimental Procedure:}

For each of the examples presented, the scheduling was performed using the same assumptions as the original reference. They are listed at the beginning of each subsection. The results were obtained without any fine tuning of the algorithm to the examples. The CPU execution times given are for a XEROX 1108 Lisp machine. This is a single-user workstation in the medium-low performance range (by today's standards).

\section{1 EXAMPLE FROM MAHA}

This example was presented in [2] and makes uses of the CSSP program [3] for the inftial stage partitioning. Two results are given in the paper, the fastest allocation and the cheapest one. For the fastest allocation, the graph is partitioned into four stages (clock cycles). For the cheapest allocation, the graph is partitioned into eight stages.

\section{As sumpt tons:}

All similar operations have equal propagation delays. For the fastest allocation: the clock cycle is such that a maximum of three operations can be combined in a 31 - $^{-}$ gle cycle

For the cheapest allocation: only one operation can be performed per cycle. 

- There ts no sharing of processors executing mutually ex-
clusive operations (i.e. operations that are on either stdo of an if-then-olse statement)

- MAHA execution times are for a VAX 11/750.

The table below gives the allocation for different $t$ iming constraints. These constraints are the number of cycles (stages) and the maximum number of operations per cycle. The results given in the last of the three sets of columns are for the HAL system when mutually excibsive operations (due to conditionals) are taken into account. This is using the method described earlier in section " 4.1 BASIC SCHEDULING ALGORITHM".

\begin{tabular}{|c|c|c|c|c|c|c|c|c|}
\hline COMPONENT & \multicolumn{2}{|c|}{ MAHA } & \multicolumn{3}{|c|}{ HAL } & \multicolumn{3}{|c|}{$\begin{array}{c}\text { HAL } \\
\text { (w. mutual } \\
\text { excluston) }\end{array}$} \\
\hline $\begin{array}{c}\text { Cycles } \\
\text { Opns/Cycle } \\
\text { subtractor } \\
(-)\end{array}$ & $\begin{array}{c}8 \\
--- \\
1 \\
--- \\
(-)\end{array}$ & \begin{tabular}{c}
4 \\
--- \\
3 \\
\hdashline$(-)$ \\
$(-)$ \\
$(-)$
\end{tabular} & $\begin{array}{c}8 \\
--- \\
1 \\
--- \\
(-)\end{array}$ & $\begin{array}{c}4 \\
--- \\
2 \\
--- \\
(-) \\
(-)\end{array}$ & $\begin{array}{c}3 \\
-\cdots \\
3 \\
--- \\
(-) \\
(-) \\
(-)\end{array}$ & $\begin{array}{c}8 \\
--- \\
1 \\
---\end{array}$ & $\begin{array}{c}4 \\
--- \\
2 \\
--- \\
(-)\end{array}$ & $\begin{array}{c}3 \\
--- \\
3 \\
(--) \\
(-)\end{array}$ \\
\hline $\begin{array}{c}\text { adder } \\
(+)\end{array}$ & $\begin{array}{l}(+) \\
--- \\
160\end{array}$ & $\begin{array}{c}(+) \\
(+) \\
--- \\
160\end{array}$ & $\frac{(+)}{--}$ & $\begin{array}{l}(+) \\
(+) \\
-- \\
25\end{array}$ & $\begin{array}{l}(+) \\
(+) \\
--- \\
35\end{array}$ & $\begin{array}{l}(+) \\
--- \\
85\end{array}$ & $\begin{array}{c}(+) \\
(+) \\
-5 \\
55\end{array}$ & $\begin{array}{r}(+) \\
(+) \\
--- \\
75\end{array}$ \\
\hline
\end{tabular}

Figure 10. Example from MAHA[2].

For the cheapest allocation (8 cycles, 1 Opn/cycle). the HAL system arrives at the same number of processors as the MAHA system. This was expected (hoped for) as all processors have $100 \%$ utilization.

For MAHA's fastest allocation ( 4 cycles, 3 Opns/cycle), the HAL system allocates one subtractor less, but with a tighter constraint on the clock cycle (2 opns/cycle). In this case the reduction in area (wtth respect to MAHA) is close to $20 \%$ with $25-30 \%$ reduction of the clock cycle time (the actual value will depend on the latch propagation delays). Processor utilizations are 100\%.

The fastest realization in the HAL system runs in three cycles ( 3 opns/cycle) and uses three adders and three subtractors. This solution requires nearly $20 \%$ more area than MAHA's fastest realization but the number of required cycles is reduced by $33 \%$

Taking mutual exclusion into account yields the most impressive results by far (given in the third set of columns). This example certainly illustrates the potential importance of doing so.

\section{2 PIPELINED FIR FILTER FROM SEHWA}

The results presented here are for the pipelined 16-point digital FIR filter example borrowed from [15]. The SEHWA system described in this paper does scheduling and allocation of pipelined data paths.

Pipeline scheduling is achleved in the HAL system by folding the DGs across the boundaries determined by the latency value (in cycles). This will cause the force-directed scheduling algortthm to balance the distribution of concurrent pipeline stages. For lack of space, we must defer the detailed explanations to a forthcoming paper [16].

\section{Assumptions:}

- The maximum stage time limit is $100 \mathrm{~ns}$

- The latency is equal to $300 \mathrm{~ns}$.

Additions are performed by adders with a delay time of 40 ns.

- Multiplications are performed by multipliers in 80 nsec

- The latch delays are $20 \mathrm{~ns}$

- Multiple succeeding operations in a single cycle are allowed.

In Figure 11 we present the results for three scheduling methods:

1. SEHWA: Backward feasible scheduling

2. SEHWA: Exhaust tve feas tble scheduling.

3. HAL: Force-directed pipel ine scheduiting.
These include the order of complexity of the algorithm, the number of cycles required, the number and type of processors a l located. and the CPU time. Only an approximate run time (on a VAX/750) was given in [15].

\begin{tabular}{|c|c|c|c|}
\hline SYSTEM & \multicolumn{2}{|c|}{ SEHWA } & HAL \\
\hline A1goritinm & Feas tble & Exhaust ive & Force-Directed \\
\hline Complexity & $o\left(n^{2} \log n\right)$ & $O\left(n^{n+2}\right)$ & $o\left(n^{2}\right)$ \\
\hline $\begin{array}{c}\text { Number of } \\
\text { Cycles }\end{array}$ & 7 & 6 & 6 \\
\hline $\begin{array}{l}\text { Number of } \\
\text { Adders }\end{array}$ & 6 & 5 & 5 \\
\hline $\begin{array}{l}\text { Number of } \\
\text { Multipl iers }\end{array}$ & 3 & 3 & 3 \\
\hline CPU time & $n / a$ & $<1$ hour & $30 \mathrm{sec}$ \\
\hline
\end{tabular}

Figure 11. FIR filter results for SEHWA Example

We see that the force-directed pipel the scheduling technique yields a result similar to that obtained by the exhaustive scheduling (i.e. an optimal result).

Futhermore, the result obtained with the backward feasibie scheduling is more expensive ( $t \times$ vs five adders) and slower (seven vs six clock cycles) although it's complexity is actually higher than that of the force-directed algor thm.

\section{3 TEMPERATURE CONTROLLER FROM ELF}

In this paper, the system was used to find the minimum number of clock cycles required given specified timing and hardware constraints. Two examples where given.

\section{Assumptions for the first example:}

- The operations of the DFG where divided into two groups with two different timing constraints.

- An initial processor library is given. It contains adders, subtractors, a comparator. a shifter (for division) and an Alu.

- The solution must not require mare than one processor of each type.

- All operations (except divisions) have a one cycle propagation delay.

- Division operations require a two cycle delay.

- succeeding (data dependent) operations cannot be performed in the same clock cycle.

- The inputs are made available sequentially (i.e. one input is made avallable in each of the first five cycles).

The Elf system generated a solution that required a total of 20 clock cycles, 12 cycles for the first group of operations and 8 for the second. In this version of the Elf system. operations in the second group cannot overlap with operations in the first. The current (unpublished) version does not have this constraint.

Given the same constraints, the HAL system arrived at a solution that required a total of 19 cycles $(1+$ clock cycles for the first group and 8 for the second). Moreover, if for the first group and 8 for the second). Moreover, if operations in both groups are allowed to overlap, but with-
out violating the separate constraints, the system arrives at solution requiring a total of 15 cycles. It can be shown that these results are optimal, given the assumptions 1 isted above.

\section{Assumptions for the second example:}

The assumptions here are the same as above except that a user-defined Alu (performing add, subtract and shift operations) is to be used. Once again, the solution must not require more than one processor of each type.

The number of cycles required by the Elf system was not specified. The HAL system scheduled the operations of the first and second groups into 13 and 8 cycles respectively. These are also optimal results. Moreover, with overlap. the These are also opt imal results. Moreover, with overlap. the
total time required is st 11115 cycles, with all constraints total time required is st 11115 cycles, with all constraints
respected. These results are mostly due to the optimal use of the Alu through the use of the multiple operation DGs described in "4.2 REFINED SCHEDULING ALGORITHM". 
The table of Figure 12 below gives the number of cycles required by both systems for the examples described. The second total number of cycles given (in the HAL column only) corresponds to the case where operations of both groups are al lowed to overlap.

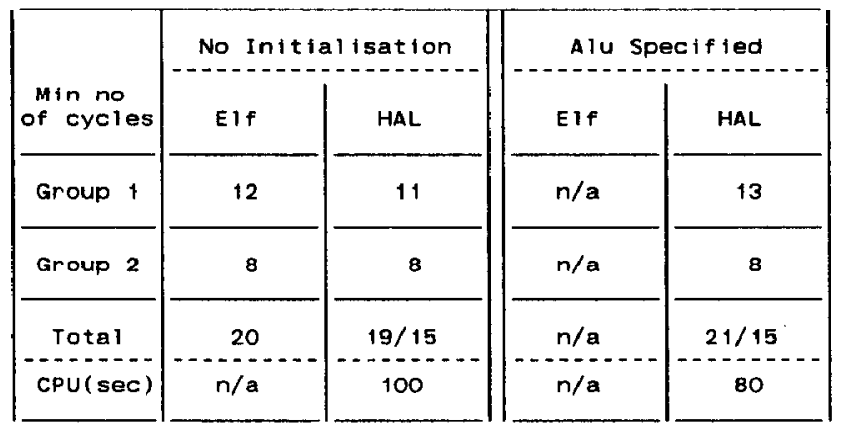

Figure 12. Minimum number of clock cycles required fordifferent hardware constratnts : Elf temperature controller example.

\subsection{FIFTH-ORDER DIGITAL 'WAVE' FILTER}

The DFG used in this example is borrowed from [11] and $1 \mathrm{~m}-$ plements a fifth-order wave digital elliptic filter. This is a much more substantial example that contains 43 operat lons (additions and multiplications) submitted to over 60 precedence constraints. It is assumed that additions require five clock cycles and multiplications ten. They were quire five clock cycles and multiplications ten. They were path is thus 17 c-steps long ( 85 cycles).

The table shown in figure 13 summarizes the allocation of processors for the example. In the last row the CPU execution time is given in minutes (again for a XERoX 1108 Lisp machine). The $17 \mathrm{c}$-step result ( 3 multipliers and 3 adders) is to be compared with that given in [11] (4 multipliers and 4 adders). Moreover, the flexiblifty of the system allows to explore alternate speed-cost trade-offs

The 21 c-step result ( 1 multiplier and 2 adders) is a good example of a substantial area saving (over 50\%) against a small loss in speed (iess than 20\%) as compared to the 17 c-step result. This area saving is even greater when comc-step result. This area saving is oven great

\begin{tabular}{|c|c|c|c|c|c|c|}
\hline COMPONENT & {$\left[\begin{array}{lll}1 & 1 & 1\end{array}\right]$} & \multicolumn{5}{|c|}{ HAL } \\
\hline $\begin{array}{c}\text { No Cycles } \\
\text { adder } \\
(+)\end{array}$ & $\begin{array}{c}17 \\
---- \\
(+) \\
(+) \\
(+) \\
(+)\end{array}$ & $\begin{array}{l}17 \\
--- \\
(+) \\
(+) \\
(+)\end{array}$ & $\begin{array}{l}18 \\
--- \\
(+) \\
(+) \\
(+)\end{array}$ & $\begin{array}{l}19 \\
-\cdots \\
(+) \\
1+)\end{array}$ & $\begin{array}{l}20 \\
\ldots . \\
(+) \\
(+)\end{array}$ & $\begin{array}{l}21 \\
\cdots- \\
\\
(+) \\
1+1\end{array}$ \\
\hline $\begin{array}{l}\text { multiplier } \\
(*) \\
\text { CPU (min) }\end{array}$ & $\begin{array}{l}(*) \\
(*) \\
(*) \\
(*) \\
--\cdot \\
n / a\end{array}$ & $\begin{array}{l}(*) \\
(*) \\
(*) \\
--- \\
1\end{array}$ & \begin{tabular}{l}
$(*)$ \\
$(*)$ \\
\hdashline 3
\end{tabular} & $\begin{array}{c}(*) \\
(*) \\
77\end{array}$ & \begin{tabular}{l}
$(*)$ \\
$(*)$ \\
\hdashline 10
\end{tabular} & \begin{tabular}{c}
$(*)$ \\
\hdashline 13
\end{tabular} \\
\hline
\end{tabular}

Figure 13. Processor allocation for all time constraints: oigital wave filter example.

This example is eloquent proof that the system can solve relatively difficult scheduling problems in a reasonable relatively difficult scheduling problems in a reasonable that all but one of the solutions found are guaranteed opt 1 mal. Exhaustive scheduling would be needed to determine if this is also the case for the rematning one.

Our experlence is that it is nearly imposslble to solve this problem manually and obtain results of the same quality. This is mostly due to the large number of interactions between operations (1.e. 43 operations submitted to over 60 tween operations (1.e. 43 operations submitted to over 60
precedence constraints). This probiem is compounded by the fact that the number of possible schedules grows exponentially with the number of cycles allowed.

On the other hand, the processing time grows linearly with the number of cycles; the complexity of the algorithm is the number of cycles; the complexity of the algor ithm is of al1 time frames.

Paper 12.1

202

\section{CONCLUSION}

The methodology presented features a novel force-directed scheduling algor thm that is invoked in a four phase scheduling/allocation scheme. We have shown how this ap proach makes it feastble to incorporate explicit allocation information to optimize the scheduling process. The information transfer is made possible by stepwise refinement of the scheduling and allocation tasks.

The force-directed scheduling algorithm at the heart of this process was shown to run in $n^{2}$ time. In sptte of this relatively low complexity, the algorithm explores the search space in a global fashion and produced optimal results for nearly all of the examples presented.

Results obtained where shown to be as good or better as the ones previously publ ished. Furthermore, the flexibility of the system was highlighted by the variety of constraints and requirements it had to deal with. These include:

Multi-cycle operations.

Multiple operations per cycle.

Mutually exclusive operations.

Pipelined data flow graphs.

Optimized use of single and multi-function processors (Alus)

Optimization of required hardware for a specified time constraint.

- Optimization of system speed for spectfled hardware resources.

\section{REFERENCES}

1. D.D. Gajski, N.D. Dutt and B.M. Pangrle, "Stilcon Comptlation (Tutorial)". Proceedings of the IEEE 1986 Custom Integrated Circuits Conference, Rochester NY. May 1986. pp. $102-110$.

2. A.C. Parker et al "MAHA: A Program for Datapath synthesis", Proceedings of the 23rd DAC (Design Automation Conference), Las Vegas. July 1986, pp. 461-466.

3. N. Park and A.C. Parker, "Synthes is of Optimal clocking Schemes", Proceedings of the 22nd DAC, July 1985, PP. 489-495.

4. E.F. Girczyc and J.P. Knight, "An ADA to Standard Cell Hardware Compiter Based on Graph Grammars and Schedul ing", Proc. of the IEEE International Conference on Computer Design (ICCD), October 1984, pp. 726-731.

5. P.G. Paulin, J.P. Knight, E.F. Girczyc, "HAL: A MuittiParadigm Approach to Automatic Data Path Synthesis" Proceedings of the 23rd DAC, July 1986, pp. 263-270.

6. P.G. Paulin, J.P. Knight, "Extended Design-Space Exploration in Automatic Data Path Synthes is". Proceedings of the 1986 Canadian Conference on VLSI, DCtober 1986, pp. $221-226$

7. T. Blackman et al, "The Stlc Silifon Complier: Language and Features", proceedings of the IEEE 22nd DAC, June 1985, pp. 232-237.

8. C. Tseng. D.P. Stewtorek, "Automated synthests of Data Paths in Digital Systems", IEEE Transactions on CAD. Juty 1986 , pp. 379-395.

9. S. Davidson et al. "Some Experiments in Local Microcode Compaction for Horizontal Machines", IEEE Transactions on Computers, July 1981 , pp. 460-477.

10. P. Marwedel, "A New Synthes is Algorithm for the MIMOLA Software System", Proceedings of the 23rd DAC, Las Vegas, July 1986, pp. 271-277.

11. S.Y. Kung, H.J. Whitehouse, T. Katlath, "VLSI and Modern Signal Processing", Prentice Hal1, 4985, pp.258-264.

12. J Nestor. D.E. Thomas, "Behavioral Synthests with Interfaces". Proceedings of the IEEE ICCAD-86 (International Conference on CAO), November 1986, PP. 112-115.

13. B.M. Pangrle, D.D. Gajski, "State Synthesfs and Connectivity Binding for Microarchitecture Compilation". Proceedings of the IEEE ICCAD-86, November 1986, pp. 210-213.

14. M.C. McFarland, "BUD: Bottom-Up Design of Digital Systems". Proceedings of the 23rd DAC. Las Vegas, July 1986. pp. 474-479.

15. N. Park, A.C. Parker, "SEHWA; A Program for Synthes is of Pipelines". Proceedings of the 23rd DAC, Las vegas, July 1986, pp. 454-460

16. P.G. Paulin, J.P. Knight, "Scheduling and Allocation for Pipelined ASICs". Submitted to the IEEE International Conference on Computer Design (ICCD '87).

Awatting ecceptance.

17. H.Trickey, "Flame1: A High-Level Hardware Comptlem", IEEE Transactions on CAD, March 1987, Pp.259-269. 